\title{
How to Identify Latent Systolic Dysfunction and Post Operative Risk in Patients with Mitral Incompetence and Normal Ejection Fraction?
}

\author{
Galal Eldin Nagib Elkilany ${ }^{1}$, Maryam De Groef ${ }^{2}$, Ibrahim Kabbash ${ }^{3}$ \\ ${ }^{1}$ Adult Cardiology Department, Medical School, Tanta University, Tanta, Egypt \\ ${ }^{2}$ SOAS, London, UK \\ ${ }^{3}$ Public Health and Statistics, Tanta, Egypt \\ E-mail: galal@kilany.org \\ Received October 25, 2011; revised November 27, 2011; accepted December 6, 2011
}

\begin{abstract}
Purpose: To study the significance of impaired positive peak rate of left ventricular (LV) pressure development $(\mathrm{MR}+\mathrm{dp} / \mathrm{dt})$ and global systolic strain (GLPSS) values in patients presented with significant mitral incompetence (MR) in coronary artery disease (CAD) and early dilated cardiomyopathy (DCM) with normal ejection fraction (EF). Methods: A description of LV contractile behavior requires measurement of the ability of the ventricle to develop force (pressure) and to shorten. Hence, performance of the ventricle as a pump assessed in the present study by measuring the pressure developed by the ventricle (Left ventricular $+\mathrm{dP} / \mathrm{dt}$ is estimated from MR jet as the rate of pressure rise from 1 to $3 \mathrm{~m} / \mathrm{sec}$ ) and shortening assessed by GLPSS (this Doppler technology allowed measurement of LV systolic strain for the entire length of LV myocardium). GLPSS and MR $+\mathrm{dp} / \mathrm{dt}$ were calculated in 30 consecutive patients (mean age was $55 \pm 12$ years) characterized by echocardiographic evidence of moderate or severe MR (in CAD and DCM patients) and normal EF (mean LV Ejection Fraction of 50.9\% $\pm 5.9 \%$ ) and compared with those obtained in 35 consecutive controls (age $54.7 \pm 11.4$ years) with normal echocardiographic study of the heart. Results: The mean values of MR $+\mathrm{dp} / \mathrm{dt}$ and GLPSS averaged from the 3 apical views, differed significantly in DCM and CAD patients (characterized by significant MR with normal EF) compared with control group, $(\mathrm{MR}+\mathrm{dp} / \mathrm{dt}=733 \pm 170$ $\mathrm{mmhg} / \mathrm{s}$ and GLPSS $-13 \% \pm 1.3 \%)$ versus $(1420 \pm 210 \mathrm{mmhg} / \mathrm{s}$ and $-19.5 \% \pm 3.3 \%)$ for patients versus control, respectively, $p<0.001$. A depressed values of MR $+\mathrm{dp} / \mathrm{dt}$ were highly correlated with GLPSS values in patients with CAD and DCM, $r=0.78$. The combined use of 2D Strain $(<-13 \%)$ and MR $\mathrm{dp} / \mathrm{dt}(<900$ $\mathrm{mmhg} / \mathrm{s}$ ) in the presence of MR (grade II or more) had $89 \%$ sensitivity and $92 \%$ specificity for detection of patients at risk of post-operative major cardiac events after MR and coronary artery bypass surgery. Conclusions: Latent LV systolic dysfunction could be defined noninvasively by depressed peak MR $+\mathrm{dp} / \mathrm{dt}$ and GLPSS in the echocardiography laboratories.
\end{abstract}

Keywords: Mitral Incompetence, Systolic Dysfunction, MR dp/dt, Global Strain

\section{Introduction}

The most commonly used index of LV contractile function is the EF which represents volume strain (change in volume divided by initial volume). However, the EF is influenced by acute or short-term as well as chronic alterations in preload, afterload and contractility. A full assessment of the contractile behavior of the ventricle requires the combined use of indices that reflect LV systolic performance, function, and contractility, as well as a consideration of global and regional function [1].

Load-sensitive measures of systolic function, including fractional area change, fractional shortening, ejection fraction, and positive peak rate of LV pressure development. Load-insensitive measures of systolic function, including the preload recruitable stroke work relationship and the end-systolic P-V relationship. Regional wall motion assessed by circumferential shortening velocity suggested enhanced circumferential fiber contractility in $\mathrm{LVH}$, but tissue Doppler imaging, used to assess longitudinal func- 
tion, was not different between controls and LVH groups. LV hypertrophy (LVH) represents the commonest condition whereby load-sensitive measures of contractility can be misinterpreted [2]. The contraction of excess sarcomeres may mask subnormal function of cardiomyocytes in the presence of LVH by maintaining normal wall shortening and thickening values. Furthermore, radial and circumferential fiber contractility is preserved in LVH, but longitudinal fiber function may be impaired, possibly due to the effects of subendocardial ischemia [3].

We hypothesized that load-sensitive methods of assessing cardiac contractility, which rely on circumferential or radial fiber function, such as the ejection fraction (EF), fractional shortening (FS), fractional area change (FAC), and the myocardial velocity of LV circumferential shortening $\left(V_{\mathrm{cf}}\right)$, may overestimate cardiac contractility in the presence of mitral incompetence and that loadinsensitive contractility indexes derived from Global Strain and MR dp/dt (rate of pressure development) analysis would better reflect and predict cardiac contractility at the cardiac myocyte level.

\section{Subjects}

Of the 65 individuals evaluated, 30 patients had significant MR allowing dp/dt measurement and were included in this study (ischaemic 18 and non-ischaemic 12). Most patients were in New York Heart Association Class II $(69 \%)$ and the remaining were class III NYHA. 35 served as control.

\section{Methods}

A description of $\mathrm{LV}$ contractile behavior requires measurement of the ability of the ventricle to develop force (pressure) and to shorten. Hence, performance of the ventricle as a pump assessed in the present study by measuring the pressure developed by the ventricle (Left ventricular $+\mathrm{dp} / \mathrm{dt}$ is estimated from MR jet as the rate of pressure rise from 1 to $3 \mathrm{~m} / \mathrm{sec}$ ) and shortening assessed by GLPSS (this Doppler technology allowed measurement of LV systolic strain for the entire length of LV myocardium which is normalized and do not require correction for LV size). GLPSS and MR $+\mathrm{dp} / \mathrm{dt}$ were calculated in 30 consecutive patients (mean age was $55 \pm 12$ years) characterized by echocardiographic evidence of moderate or severe MR (in CAD and DCM patients) and normal EF (mean LV Ejection Fraction of $50.9 \% \pm 5.9 \%$ ) and compared with those obtained in 35 consecutive controls (age $54.7 \pm 11.4$ years) with normal echocardiographic study of the heart Figure 1.

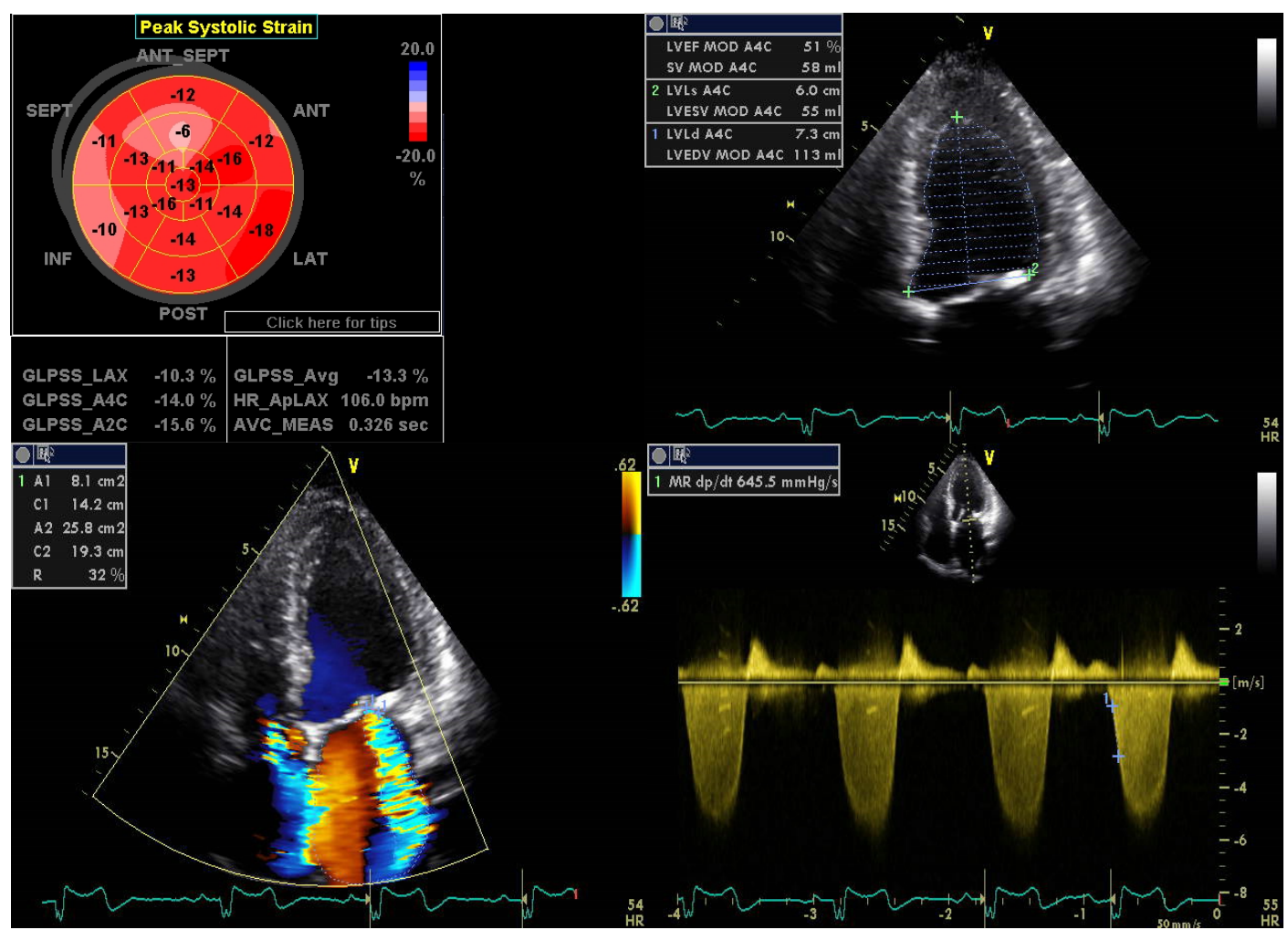

Figure 1. Bulls eye technique (automated function imaging algorithm) generated from apical 2,3 and 4 chamber views illustrated the impaired global longitudinal peak systolic strain (GLPSS) of -13\% in patient with significant mitral incompetence but normal ejection fraction $(\mathrm{EF}=51 \%)$, note that : $\mathrm{MR}+\mathrm{dp} / \mathrm{dt}=645 \mathrm{mmhg} / \mathrm{s}$ only. 
The automated function imaging (AFI) algorithm noninvasively tracks and analyzes peak systolic strain based on 2D strain: Digital loops were acquired from apical 2-, 3 - and 4-chamber views and a line was traced along the LV endocardium (Figure 1). Around this line, the software selected natural acoustic markers moving with the tissues automatic frame by frame tracking of these markers during the heart cycle yielded a measure of contractility along the selected region of interest.

GLPSS and MR + dp/dt were calculated in 30 consecutive patients (mean age was $55 \pm 12$ years) (Figures 2-3).

\section{Statistics Used}

The collected data were tabulated and statistically analyzed using SPSS software statistical package version 17.

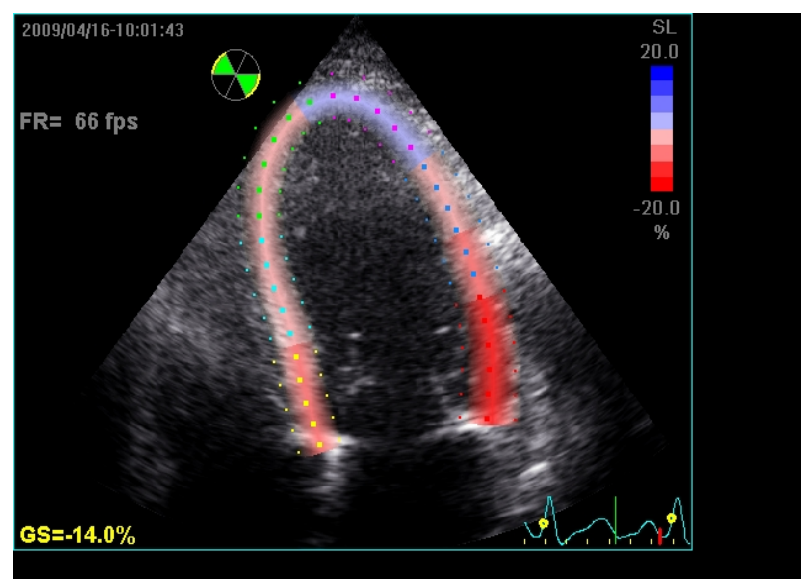

Figure 2. Global Longitudinal Peak Systolic Strain in 4 chamber view is below normal values $(-14 \%)$ inspite of normal EF by 2 Dimensional echocardiography [Biplane Technique].

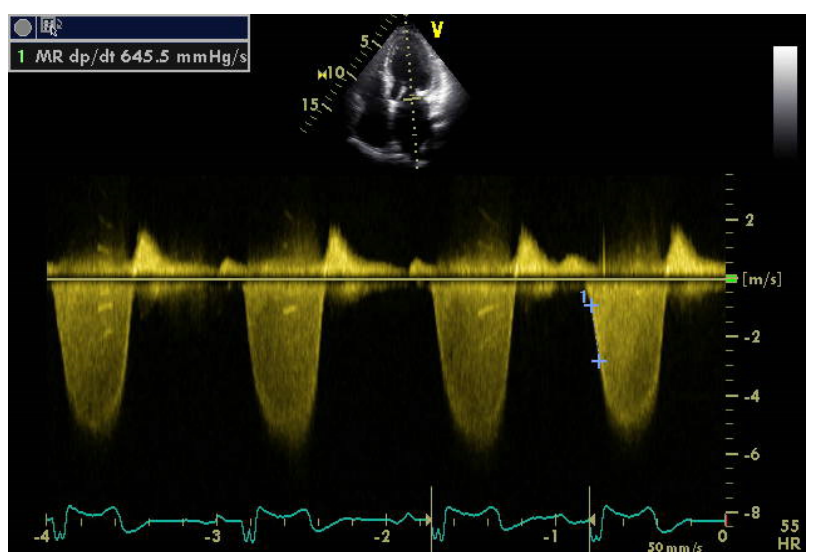

Figure 3. Left ventricular $\mathrm{dp} / \mathrm{dt}$ is estimated from mitral regurgitation (MR) jet as the rate of pressure rise (RPR) from 1 to $3 \mathrm{~m} / \mathrm{sec}$. Isometric Contraction was estimated as pre-ejection time minus Q-first sound (S1).
For quantitative variables, mean and standard deviation were calculated. Because of small sample size of each studied categories, non parametric tests of significance were used. Kruskal-Wallis test was used to compare between mean values of studied groups if they were more than two and Mann-Whitney test to compare two groups. Wilcoxon singed rank test was used to compare mean values of $\mathrm{EF} \%$ before and after the intervention. Categorical variables were represented as number and percentage and Monte Carlo test was used as test of significance. The level of significance was adopted at $p<0.05$.

\section{Results}

The mean values of MR $+\mathrm{dp} / \mathrm{dt}$ and GLPSS averaged from the 3 apical views, differed significantly in DCM and CAD patients (characterized by significant MR with normal EF) compared with control group, $(\mathrm{MR}+\mathrm{dp} / \mathrm{dt}=$ $733 \pm 170 \mathrm{mmhg} / \mathrm{s}$ and GLPSS $-13 \% \pm 1.3 \%$ ) versus $(1420 \pm 210 \mathrm{mmhg} / \mathrm{s}$ and $-19.5 \% \pm 3.3 \%)$ for patients versus control, respectively, $p<0.001$.

The combined use of 2D Strain $(<-13 \%)$ and MR dp/dt $(<900 \mathrm{mmhg} / \mathrm{s})$ in the presence of MR (>grade II/IV) had $89 \%$ sensitivity and $92 \%$ specificity for detection of patients at risk of post-operative major cardiac events following mitral repair and/or coronary artery bypass surgery (Figure 4).

\section{6. dp/dt}

After cardiac surgery, EF and $+\mathrm{dp} / \mathrm{dt}$ were significantly improved $(p<0.001)$ in the group of patients characterized by an average pre operative value of $\mathrm{dp} / \mathrm{dt}>900$ $\mathrm{mmhg} / \mathrm{s}$ overall, with no difference between the ischemic and non-ischemic groups (Figure 5).

However, a depressed values of MR $+\mathrm{dp} / \mathrm{dt}$ pre operatively were highly correlated with post operative depression of EF in patients with CAD and DCM, $\mathrm{r}=0.790, p$ $=0.001$, Figure 6 .

\section{Global Logitudinal Peak Systolic Strain (GLPSS)}

Global strain was calculated as the average of maximum strain in all LV segments. in order to test if global strain could express the ideal LV systolic function in comparison with 2 dimensional \& real time 3 dimensional echocardiography. GLPSS averaged from the 3 apical views (Bulls-eye display), differed significantly in patients with left ventricular systolic dysfunction (depressed values of $\mathrm{dp} / \mathrm{dt}$ in DCM and ischemic MR) compared with control patients (GLPSS $-13 \%$ ) versus $(-19.5 \%)$ for patients vs control, respectively, $(p<0.0001)$ (Figure 7). 
Blue line: GLPSS $<-13 \% \&$

$\mathrm{MR}+\mathrm{dp} / \mathrm{dt}<900 \mathrm{mmhg} / \mathrm{s}$,

Green line represent GLPSS $>-13 \% \& \mathrm{MR}+\mathrm{dp} / \mathrm{dt}>900 \mathrm{mmhg} / \mathrm{s}$

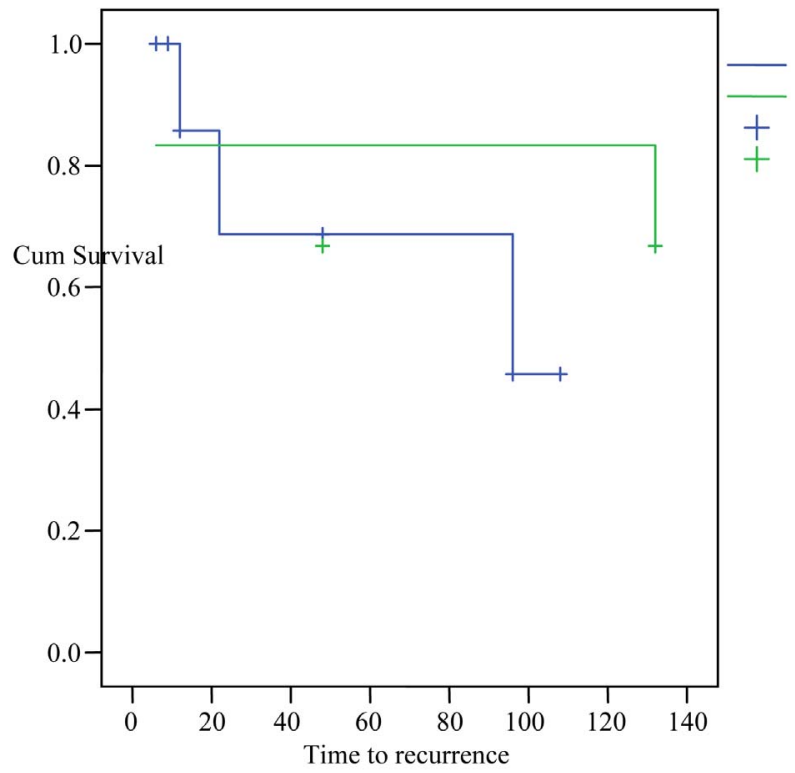

Figure 4. Recipient-observer characteristic curve: showed high incidence of major cardiac events (cardiac death included) among patients with significant mitral incompetence and depression of Global strain (GLPSS) as well as low MR + dp/dt values. Note that: patients represented by blue line who have pre operative low global systolic strain and depressed dp/dt values Showed severe depression of cardiac function (EF $<25 \%$ ) and high incidence of major cardiac events post operatively on 140 days follow up (inspite of normal EF pre operatively).
In intermediate term follow up (140 days), a depressed values of GLPSS had $89 \%$ sensitivity and 92 specificity for prediction of patients prone to develop severe LV systolic dysfunction (EF of $\leq 30 \%$ - 35\%) post operatively and patients at risk for major cardiac events (cardiac death, acute heart failure, pumonary edema, frequent hospitalization for progressive heart failure symptoms, need for cardiac re synchronization therapy, revascularization, and/or need for cardiac transplantation).

\section{Discussion}

Our study shows for the first time that echocardiographic assessment of Global Strain and MR $+d p / d t$ is a useful predictor of Intermediate-term clinical outcome in ischemic and non-ischemic mitral incompetence.

This is consistent partly with previous work from Oguz E. et al., showing that LV dp/dt-rise was the single variable significantly different between CRT (cardiac resynchronization therapy) responders and non-responders (defined by the change in NYHA-class) [4].

Moreover, there is limited information regarding whether an acute hemodynamic response to CRT (assessed as percent change of the baseline $\mathrm{dp} / \mathrm{dt}$ ) is associated with a favorable clinical course, and whether there are cardiac substrate specific outcome in ischemic and non-ischemic cardiomyopathy. However, recent work has indicated that acute beneficial hemodynamic changes are sustained at a 6-month follow-up [5].

Furthermore, Tournoux et al., concluded that acute in-

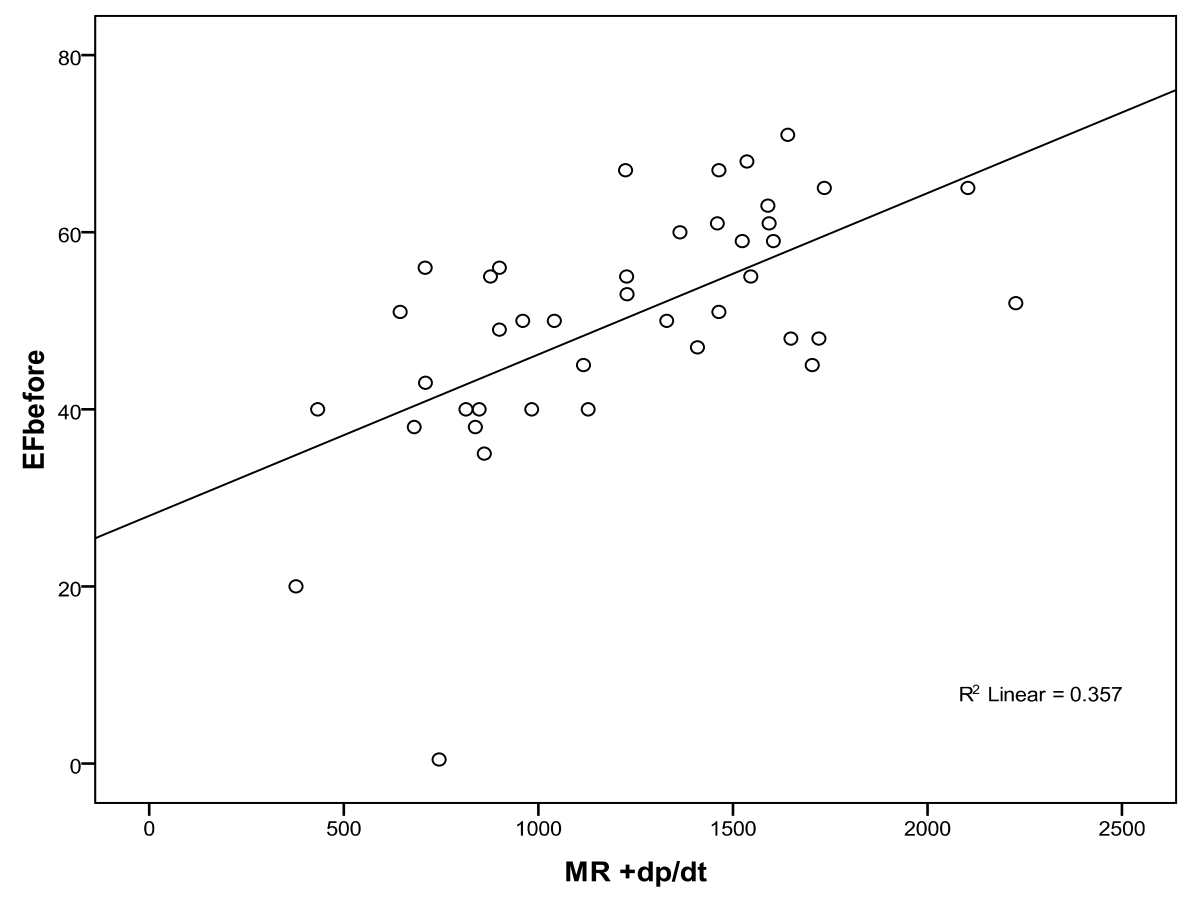

Figure 5. Correlation between $\mathbf{E F} \%$ before intervention and $\mathrm{dp} / \mathrm{dt}, \mathrm{r}=\mathbf{0 . 5 9 8}, \boldsymbol{p}=\mathbf{0 . 0 0 1}$. 


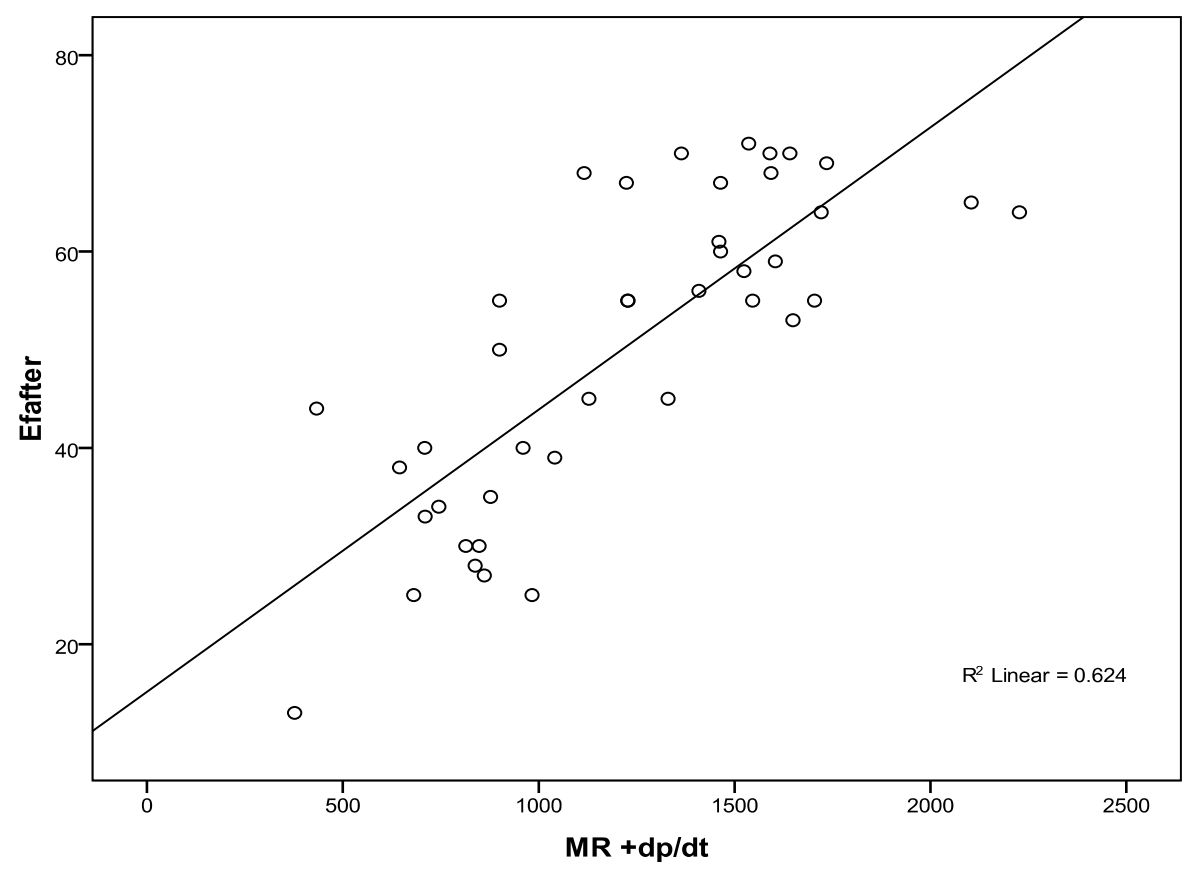

Figure 6. Correlation between $\mathrm{EF} \%$ after intervention and $\mathrm{dp} / \mathrm{dt}, \mathrm{r}=\mathbf{0 . 7 9 0 , p}=\mathbf{0 . 0 0 1}$.

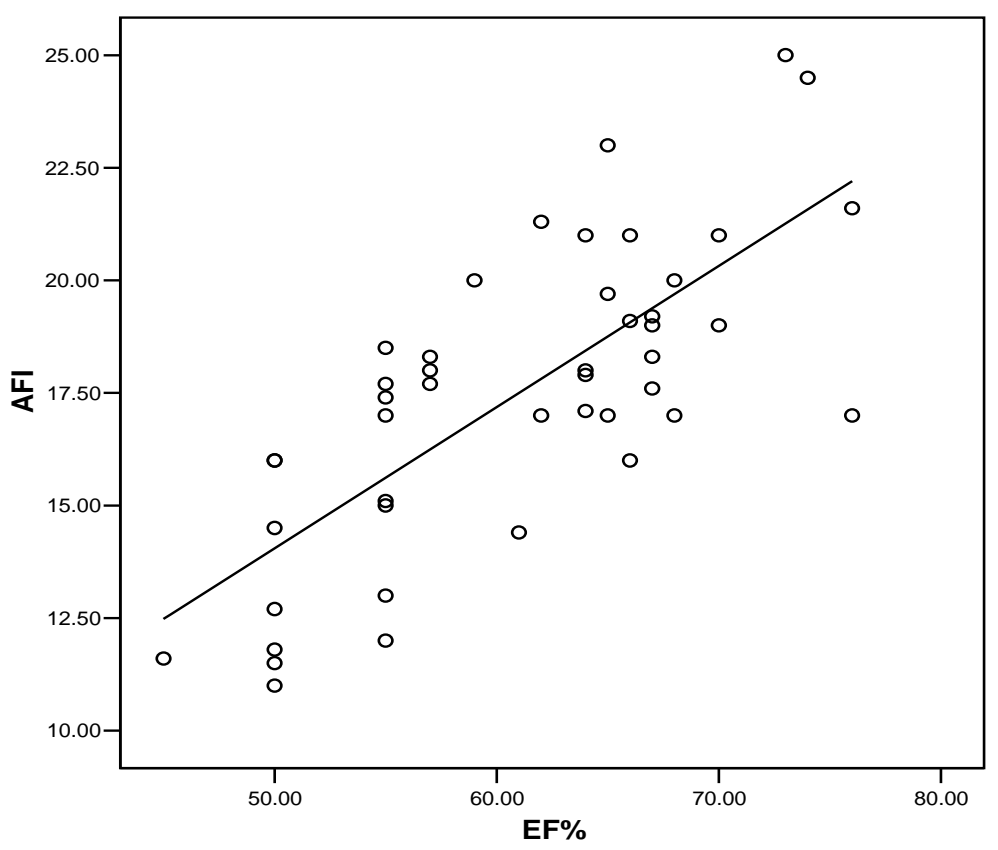

Figure 7. Correlation between EF\% and AFI (GLPSS) among studied control.

crease in LV dp/dt $(\Delta \mathrm{dp} / \mathrm{dt})$ by Doppler echocardiography is a relatively simple non-invasive test for the acute haemodynamic response after CRT implantation.

In the Cox proportional hazard model, change in $\mathrm{dp} / \mathrm{dt}$ was the only significant predictor of event-free survival after adjustment (HR 1/4 0.95 per unit dp/dt change, $\mathrm{P}$ $1 / 40.0176)$. Other clinical variables kept in the model (serum creatinine, etiology of the cardiomyopathy, per- cent change in EF acutely after CRT, and percent change in ESV acutely after CRT), were not significant predictors after adjustment [6].

The Previous works concluded that ventricular contractility as measured by $\mathrm{dp} / \mathrm{dt} \max$ is a predictor of outcome in patients with heart failure [7].

In the present study, MR $+\mathrm{dp} / \mathrm{dt}$ and Global Strain (utilizing automated function imaging algorithm) were 
calculated in 65 subjects ( 30 patients and 35 controls) and EF measured by 2 and 3 dimensional echocardiography [biplane and tri-plane geometry], providing a more accurate measure of the extent of global and regional dysfunction of the left ventricle even in the presence of apparently normal ejection fraction.

Acute and intermediate-term hemodynamic improvement due to revascularization or/and mitral valve surgery were correlated to basic pre operative normal values of $\mathrm{dp} / \mathrm{dt}$ and minimally reduced GLPSS values, with minimal chance for time-dependent changes in factors such as pre-load to confound the analysis.

In this study, measurements of Global peak systolic strain had excellent correlation with LVEF (derived from $2 \& 3$ dimensional echocardiography) and of great help in identifying patients at risk of major cardiac events when rate of pressure development was also depressed. This observation has important diagnostic and therapeutic implications and lead to improvement in non invasive risk stratification for individual patients with significant mitral incompetence and borderline LVEF.

In accordance with our study, a recent study conducted by Edvardsen, revealed that: Global strain correlated well with total infarct size; a global strain value less than $-11.7 \%$ in absolute values had very good sensitivity and specificity to predect infarct size including more than $18 \%$ of the total LV [8].

Indeed, in CAD patients LVEF showed a poor correlation to infarct size by MRI. One possible reason is that LVEF describes the global LV function, where as the infarcted area and reduced function is regional. A decrease in LVEF is supposes that several LV segments or large area of infarcted myocardium are involved. Methods that measure LV regional function could therefore be more sensitive measures than EF to identify systolic dysfunction, and the assessment of myocardial strain has been shown to be superior to wall motion analysis in CAD and is fundamentally a regional technique [9].

In mitral valve incompetence, measures such as ejection fraction (EF) are load-dependent and the increased preload may enhance EF despite a subtle decrease in contractility. By the time EF is low, there may already have been irreversible myocardial damage [10].

In addition, in the pathophysiology of heart failure, exercise echocardiography reveals complex abnormalities of both systolic and diastolic ventricular function Involving Torsion, Untwist, and Longitudinal Motion [11].

\section{Clinical Implication}

Although the measure used in this study was made in the immediate pre-operative period, one could speculate that such a measure made intra-procedurally could facilitate selecting an optimal patient for mitral valve surgery and CABG who are at risk of post operative major cardiac events.

Additionally, measure of dp/dt may also prove useful in selecting patients at high risk of post operative severe left ventricular systolic dysfunction and cardiac death and need for post-procedural CRT/Defibrillator device implantation.

\section{Limitation}

Technical aspects is of crucial importance in the present study; surgeon experience could affect the results positively or negatively; complete revascularization and optimal mitral valve repair surgery is not a simple procedure for all cardiac surgeon, and if done by expert hands will improve cardiac function ( $+\mathrm{dp} / \mathrm{dt} \&$ GLPSS) specially in patients who had a large ischemic although viable myocardium preoperatively.

In addition, the etiology of mitral incompetence and type of mitral valve surgery play an important role in our results.

Finally, tachyarrhthymias affect global longitudinal peak systolic strain values.

\section{References}

[1] M. K. Karunanithi and M. P. Feneley, "Single-Beat Determination of Preload Recruitable Stroke Work Relationship: Derivation and Evaluation in Conscious Dogs," Journal of the American College of Cardiology, Vol. 35, 2000, pp. 502-513. doi:10.1016/S0735-1097(99)00566-5

[2] B. A. Carabello, "Evolution of the Study of Left Ventricular Function: Everything Old Is New Again," Circulation, Vol. 105, 2002, pp. 2701-2703. doi:10.1161/01.CIR.0000021240.86593.9D

[3] N. H. Andersen and S. H. Poulsen, "Evaluation of the Longitudinal Contraction of the Left Ventricle in Normal Subjects by Doppler Tissue Tracking and Strain Rate," Journal of the American Society of Echocardiography, Vol. 16, No. 7, 2003, pp. 716-723. doi:10.1016/S0894-7317(03)00325-0

[4] E. Oguz, B. Dagdeviren, T. Bilsel, O. Akdemir, I. Erdinler, A Akyol, T. Ulufer, T. Tezel and K. Gurkan, "Echocardiographic Prediction of Long-Term Response to Biventricular Pacemaker in Severe Heart Failure," European Journal of Heart Failure, Vol. 4, No. 1, 2002, pp. 83-90. doi:10.1016/S1388-9842(01)00188-X

[5] P. Steendijk, S. A. Tulner, J. J. Bax, P. V. Oemrawsingh, G. B. Bleeker, L. van Erven, H. Putter, H. F. Verwey, E. E. van der Wall and M. J. Schalij, "Hemodynamic Effects of Long-Term Cardiac Resynchronization Therapy: Analysis by Pressure Volume Loops," Circulation, Vol. 113, 
2006, pp. 1295-1304. doi:10.1161/CIRCULATIONAHA.105.540435

[6] F. B. Tournoux, C. Alabiad, D. Fan, A. A. Chen, M. Chaput, K. Heist, T. Mela, M. Mansour, V. Reddy, J. N. Ruskin, M. H. Picard and J. P. Singh, "Echocardiographic Measures of Acute Haemodynamic Response after Cardiac Resynchronization Therapy Predict Long-Term Clinical Outcome," European Heart Journal, Vol. 28, No. 9, 2007, pp. 1143-1148. doi:10.1093/eurheartj/ehm050

[7] T. J. Kolias, K. D. Aaronson and W. F. Armstrong, "Doppler-Derived dp/dt and $2 \mathrm{dp} / \mathrm{dt}$ Predict Survival in Congestive Heart Failure," Journal of the American College of Cardiology, Vol. 36, No. 5, 2000, pp. 1594-1599. doi:10.1016/S0735-1097(00)00908-6

[8] M. D. Thor Edvardsen, "Early Prediction of Infarct Size by Strain after Coronary Perfusion, Cardiovascular Surgery 2007," ACC 9/12/2007, American College of Cardiology Foundation.
[9] T. Edvardsen, B. L. Gerber, J. Garot, D. A. Bluemke, J. A. Lima and O. A. Smiseth, "Quantitative Assessment of Intrinsic Regional Myocardial Deformation by Doppler Strain Rate Echocardiography in Humans: Validation against Three-Dimensional Tagged Magnetic Resonance Imaging," Circulation, Vol. 106, 2002, pp. 50-56. doi:10.1161/01.CIR.0000019907.77526.75

[10] J. L. Harrison and B. D. Prendergast, "Mitral Valve Repair: Benefits of Early Intervention and Future Prospects," European Journal of Cardiology, Vol. 5, No. 30 , 2007.

[11] Y. T. Tan, F. Wenselburger, E. Lee, et al., "The Pathophysiology of Heart Failure with Normal Ejection Fraction: Exercise Echocardiography Reveals Complex Abnormalities of Both Systolic and Diastolic Ventricular Function Involving Torsion, Untwist, and Longitudinal Motion," Journal of the American College of Cardiology, Vol. 54, No. 1, 2009, pp. 36-46. doi:10.1016/j.jacc.2009.03.037 